\title{
Quantum eigenstate tomography with qubit tunneling spectroscopy
}

\author{
Anatoly Yu. Smirnov and Mohammad H. Amin \\ D-Wave Systems Inc., 3033 Beta Avenue, Burnaby BC Canada V5G $4 M 9$
}

\begin{abstract}
Measurement of the energy eigenvalues (spectrum) of a multi-qubit system has recently become possible by qubit tunneling spectroscopy (QTS). In the standard QTS experiments, an incoherent probe qubit is strongly coupled to one of the qubits of the system in such a way that its incoherent tunneling rate provides information about the energy eigenvalues of the original (source) system. In this paper, we generalize QTS by coupling the probe qubit to many source qubits. We show that by properly choosing the couplings, one can perform projective measurements of the source system energy eigenstates in an arbitrary basis, thus performing quantum eigenstate tomography. As a practical example of a limited tomography, we apply our scheme to probe the eigenstates of a kink in a frustrated transverse Ising chain.
\end{abstract}

\section{INTRODUCTION}

Superconducting qubits are used as the basic building blocks for practical implementation of scalable quantum computers [1]. In particular, the existing annealingbased quantum processing units (QPU) [2] exploit flux qubits based on superconducting quantum devices (rfSQUIDs) [3, 4]. The qubits are controlled by a limited number of low-bandwidth external lines. This feature allows creating a quantum processor with more than 1000 qubits, while at the same time keeping a low level of noise in the system. Experimental technique, termed qubit tunneling spectroscopy (QTS) [5], has been developed in order to measure quantum spectra of superconducting qubits (source qubits) using probe qubits undergoing incoherent tunneling transitions. A similar idea of weakly coupling a probe qubit to the quantum system with the goal of observing its energy spectrum was proposed in Ref. [6]. In Ref. [5], however, the coupling between the probe qubit and the source qubits is strong and the method was experimentally implemented with rf-SQUID flux qubits. Quantum spectra, characterized by line splittings of the order of few $\mathrm{GHz}$, were measured using MHz-range control lines. The same technique was also employed to demonstrate quantum entanglement in systems of two and eight flux qubits embedded into an industrial-scale quantum annealing processor [7]. These experiments are performed with a unit cell having a linear size of the order of $0.3 \mathrm{~mm}$. Quantum spectra taken in the process show very well resolved spectral lines, thus demonstrating a clear example of macroscopic quantum coherence [8] in a multi-qubit system.

In QTS, information about quantum properties of the source qubits is extracted from the bias dependence of the incoherent tunneling rate, $\Gamma(\epsilon)$, of the probe qubit. Here $\epsilon$ is the external flux applied to the probe along $z$-axis. Positions of the maxima of $\Gamma(\epsilon)$ determine the energy levels of the source system, whereas its peak amplitude is proportional to the overlap between the eigenfunctions of the total (probe plus source) system before and after the tunneling [a, $[$, $]$.

In this paper, we generalize QTS technique to the case where the probe qubit is coupled to many source qubits in an arbitrary basis. We show that projective measurements of energy eigenstates of the source system in an arbitrary basis can be performed with this approach. Therefore, our measurement scheme is tomgraphically complete. As a practical example, we consider dynamics of a kink in a frustrated transverse Ising chain, in which the nearby qubits are coupled ferromagnetically and the first and the last qubits are biased in the opposite direction. The classical states with the lowest energy have a kink, which can be located between any nearby qubits. This kink behaves like a free particle confined in a potential well. We provide numerical calculations of the incoherent tunneling rate of the system taking into account the low frequency environment. The maxima of the tunneling rate, plotted as a function of the bias applied to the probe, are shown to be proportional to the modulus squared of the eigenfunctions.

\section{QUBIT TUNNELING SPECTROSCOPY}

Following Ref. [5], here and in Appendix $\mathrm{A}$ we derive a set of formulas describing multi-qubit QTS experiments. The quantum system under study has $N$ coupled qubits, with Hamiltonian $H_{S}$ written in terms of of Pauli matrices $\left\{\sigma_{i}^{x}, \sigma_{i}^{y}, \sigma_{i}^{z}\right\}$ where $i \in\{1, \ldots, N\}$. We denote eigenstates and eigenvalues of this Hamiltonian by $\left|\Psi_{n}\right\rangle$ and $E_{n}$, respectively, where $n \in\left\{0,1, \ldots,\left(2^{N}-1\right)\right\}$. There is no need to specify Hamiltonian $H_{S}$ of the quantum system at this stage, although later we will consider the transversal Ising chain as an example. In addition to the above source qubits operating in a fully quantum regime, we have one probe qubit characterized by a small tunneling amplitude $\Delta_{p}$ and by an external bias $\epsilon$. This qubit works in an incoherent regime of macroscopic resonant tunneling (MRT) [9, 10]. We write the total source-probe Hamiltonian as:

$$
H_{0}=H_{S}+\left(H_{C}+\epsilon / 2\right)\left(1-\sigma_{p}^{z}\right)-\Delta_{p} \sigma_{p}^{x}
$$

The probe qubit, which is described by the set of its Pauli matrices $\left\{\sigma_{p}^{x}, \sigma_{p}^{y}, \sigma_{p}^{z}\right\}$, has two eigenstates, $\left|\uparrow_{p}\right\rangle$ and $\left|\downarrow_{p}\right\rangle$, 
of the matrix $\sigma_{p}^{z}: \quad \sigma_{p}^{z}\left|\uparrow_{p}\right\rangle=\left|\uparrow_{p}\right\rangle, \sigma_{p}^{z}\left|\downarrow_{p}\right\rangle=-\left|\downarrow_{p}\right\rangle$. The coupling between the source qubits and the probe is provided by the term: $-\sigma_{p}^{z} H_{C}$. Once again, the details of $H_{C}$ does not affect our general description. The second term in the Hamiltonian vanishes when probe qubit is in state $\left|\uparrow_{p}\right\rangle$. Therefore, we can write

$$
H_{0}=H_{S}^{\uparrow} \otimes\left|\uparrow_{p}\right\rangle\left\langle\uparrow_{p}\left|+H_{S}^{\downarrow} \otimes\right| \downarrow_{p}\right\rangle\left\langle\downarrow_{p}\right|-\Delta_{p} \sigma_{p}^{x},
$$

where $H_{S}^{\uparrow}=H_{S}$ and $H_{S}^{\downarrow}=H_{S}+2 H_{C}+\epsilon$. We denote the eigenstates of these Hamiltonians by $\left|\Psi_{n}^{\uparrow}\right\rangle$ and $\left|\Psi_{m}^{\downarrow}\right\rangle$ :

$$
\begin{aligned}
H_{S}\left|\Psi_{n}^{\uparrow}\right\rangle & =E_{n}^{\uparrow}\left|\Psi_{n}^{\uparrow}\right\rangle, \\
H_{S}^{\downarrow}\left|\Psi_{m}^{\downarrow}\right\rangle & =\left(E_{m}^{\downarrow}+\epsilon\right)\left|\Psi_{m}^{\downarrow}\right\rangle .
\end{aligned}
$$

Notice that $\left|\Psi_{n}^{\uparrow}\right\rangle=\left|\Psi_{n}\right\rangle$ and $E_{n}^{\uparrow}=E_{n}$.

In the limit of small $\Delta_{p}$, the eigenstates of $H_{0}$ are approximately $\left|\psi_{n}^{\uparrow}\right\rangle=\left|\Psi_{n}^{\uparrow}\right\rangle \otimes\left|\uparrow_{p}\right\rangle$ and $\left|\psi_{m}^{\downarrow}\right\rangle=\left|\Psi_{m}^{\downarrow}\right\rangle \otimes\left|\downarrow_{p}\right\rangle$, where $n, m \in\left\{0,1, \ldots,\left(2^{N}-1\right)\right\}$. When the probe qubit is in its "up" state, $\left|\uparrow_{p}\right\rangle$, the eigenstates of the source qubits coincide with the eigenstates of the original Hamiltonian $H_{S}$. Notice that this is true even when coupling to the probe qubit is strong. In the opposite case, when the probe is in its "down" state, $\left|\downarrow_{p}\right\rangle$, the coupling to the probe should create a large bias for the source qubits in such a way that the new Hamiltonian, $H_{S}^{\downarrow}$, has a welldefined ground state $\left|\Psi_{0}^{\downarrow}\right\rangle$ isolated from the rest of the eigenstates by a large energy gap. The total system is then initialized in $\left|\psi_{0}^{\downarrow}\right\rangle=\left|\Psi_{0}^{\downarrow}\right\rangle \otimes\left|\downarrow_{p}\right\rangle$ and tunnels to one of the eigenstates $\left|\psi_{n}^{\uparrow}\right\rangle=\left|\Psi_{n}^{\uparrow}\right\rangle \otimes\left|\uparrow_{p}\right\rangle$, as the probe tunnels from $\left|\downarrow_{p}\right\rangle$ to $\left|\uparrow_{p}\right\rangle_{\text {. The two sets of states of the total }}$ Hamiltonian $H_{0}$, with the probe qubit being in $\left|\downarrow_{p}\right\rangle$ or $\left|\uparrow_{p}\right\rangle$, can be moved relative to each other by applying a probe bias $\epsilon$. The rate of macroscopic tunneling between the initial and final states can show well-resolved peaks when the eigenenergies of these states are in resonance [10].

To have a full description of the dynamics we consider the system being exposed to an environment. As shown in Ref. 7], the width of the MRT peaks is predominantly determined by the low frequency noise coupled to the probe qubit. As such, in our modeling we consider an environment only interacting with the probe qubit. The dissipative dynamics of the probe-source system is therefore described by the master equation (A26) written in our case as (see Appendix $\mathrm{A}$ )

$$
\dot{P}_{0}+\Gamma_{0} P_{0}=\sum_{n} \Gamma_{0 n} P_{n}
$$

where $P_{0}$ and $P_{n}$ are the probabilities of being in state $\left|\psi_{0}^{\downarrow}\right\rangle$ and $\left|\psi_{n}^{\uparrow}\right\rangle$, respectively, and

$$
\begin{array}{r}
\Gamma_{0}(\epsilon)=\Delta_{p}^{2} \sum_{n}\left|\left\langle\Psi_{n}^{\uparrow} \mid \Psi_{0}^{\downarrow}\right\rangle\right|^{2} \times \\
\sqrt{\frac{2 \pi}{W^{2}}} \exp \left[-\frac{\left(E_{n}^{\uparrow}-E_{0}^{\downarrow}-\epsilon+\epsilon_{p}\right)^{2}}{2 W^{2}}\right],
\end{array}
$$

$$
\begin{array}{r}
\Gamma_{0 n}(\epsilon)=\Delta_{p}^{2}\left|\left\langle\Psi_{n}^{\uparrow} \mid \Psi_{0}^{\downarrow}\right\rangle\right|^{2} \times \\
\sqrt{\frac{2 \pi}{W^{2}}} \exp \left[-\frac{\left(E_{n}^{\uparrow}-E_{0}^{\downarrow}-\epsilon-\epsilon_{p}\right)^{2}}{2 W^{2}}\right]
\end{array}
$$

The MRT width $W$ and the reorganization energy $\epsilon_{p}$ are related by fluctuation dissipation theorem (see Ref. [9] and Appendix A . At $t=0$, we have $P_{n}=0$, and therefore the initial slope of probability decay is only given by $\Gamma_{0}$, which is measured in the MRT experiments. At the point of resonance, where $\epsilon=E_{n}^{\uparrow}-E_{0}^{\downarrow}+\epsilon_{p}$, the peak value of the escape rate $\Gamma_{0}$ is proportional to the overlap of the initial and final wave functions,

$$
\Gamma^{\text {peak }} \propto\left|\left\langle\Psi_{n}^{\uparrow} \mid \Psi_{0}^{\downarrow}\right\rangle\right|^{2} .
$$

In QTS, the probe qubit bias, $\epsilon$, is swept within some range and $\Gamma_{0}(\epsilon)$ is measured. The locations of the peaks of $\Gamma_{0}$ give information about the energy spectrum, $E_{n}$, of the source system.

\section{QUANTUM EIGENSTATE TOMOGRAPHY}

For spectroscopy purposes, the details of $H_{C}$ are unimportant as long as the ground state of $H_{S}^{\downarrow}$ is well separated from excited states and has overlap with the eigenstates of $H_{S}^{\uparrow}\left(=H_{S}\right)$ that we want to detect. Since the tunneling rate is proportional to $\left|\left\langle\Psi_{n}^{\uparrow} \mid \Psi_{0}^{\downarrow}\right\rangle\right|^{2}$, if we can set $\left|\Psi_{0}^{\downarrow}\right\rangle$ as an arbitrary state, we can measure the projection of $\left|\Psi_{n}^{\uparrow}\right\rangle$ on that state and therefore perform tomography on the source system's eigenstates, $\left|\Psi_{n}\right\rangle$. This can be done by choosing a specific form of $H_{C}$. For example, assume that the probe qubit can be coupled to all source qubits along all three axes. We can therefore write

$$
H_{C}=\sum_{i=1}^{N}\left(J_{p i}^{x} \sigma_{i}^{x}+J_{p i}^{y} \sigma_{i}^{y}+J_{p i}^{z} \sigma_{i}^{z}\right)
$$

The coupling constants $J_{p i}^{x}, J_{p i}^{y}$ and $J_{p i}^{z}$ create additional biases for the $i$-th source qubit along $x, y$, and $z$ directions. These biases disappear in the case when the probe qubit is in $\left|\uparrow_{p}\right\rangle$ and, as before, $H_{S}^{\uparrow}=H_{S}$. In the opposite direction of the probe, $\left|\downarrow_{p}\right\rangle$, strong coupling constants $\left\{J_{p i}^{x}, J_{p i}^{y}, J_{p i}^{z}\right\}$ can make $H_{C}$ dominate in $H_{0}$, thus suppressing the contribution of $H_{S}$. The standard $z-z$ couplers [3, 4] between the probe and source qubits lead to the following set of the constants: $\left\{0,0, J_{p i}^{z}\right\}$. This set creates the $z$-directed initial state $\left|\Psi_{0}^{\downarrow}\right\rangle=\otimes_{i=1}^{N}\left|z_{i}\right\rangle$, defined in terms of the eigenstates $\left|z_{i}\right\rangle$ of the matrices $\sigma_{i}^{z}$. Again, here the probe qubit is in the $\left|\downarrow_{p}\right\rangle$ state. The up or down direction of the specific $i$-th source qubit in the reference state $\left|\Psi_{0}^{\downarrow}\right\rangle$ depends on the sign of the corresponding coupling coefficient $J_{p i}^{z}$. If instead of $z-z$ couplers we have $x$ - $z$ couplers, i.e., $J_{p i}^{x} \neq 0$ and $J_{p i}^{y}=J_{p i}^{z}=0$, then the reference state will be $\left|\Psi_{0}^{\downarrow}\right\rangle=\otimes_{i=1}^{N}\left|x_{i}\right\rangle$, where $\left|x_{i}\right\rangle$ are the eigenfunctions of $\sigma_{i}^{x}$. We can therefore project 
the eigenstates $\left|\Psi_{n}\right\rangle$ onto the $x$-basis. Likewise, we can project $\left|\Psi_{n}\right\rangle$ onto $y$-basis. Being able to do projective measurements in all basis makes our protocol tomographically complete.

In practice, the coupling of the probe qubit to all source qubits in all bases could be challenging. However, with a limited number of couplers working in a single basis we can still do projective measurements in a very limited Hilbert subspace and obtain useful information. For example, in ferromagnetic systems, one can do projective measurements of the eigenstates onto the lowest energy subspace $\{|\uparrow \uparrow \ldots \uparrow\rangle,|\downarrow \downarrow \ldots \downarrow\rangle\}$ with one coupler [7]. In the next section, we provide another example in which the coupling of the probe qubit to two source qubits is needed for projection onto the lowest energy subspace.

\section{WAVE FUNCTION OF A KINK IN THE FRUSTRATED ISING CHAIN}

As an example of the source quantum system we consider a frustrated Ising chain described by the following Hamiltonian $H_{S}$,

$$
H_{S}=\sum_{i-1}^{N}\left(h_{i} \sigma_{i}^{z}-\Delta_{i} \sigma_{i}^{x}\right)+\sum_{i<j} J_{i j} \sigma_{i}^{z} \sigma_{j}^{z} .
$$

For this specific case the transverse field is determined by the set of tunneling amplitudes $\left\{\Delta_{i}\right\}$. The qubits are biased along $z$-direction with the strengths $\left\{h_{i}\right\}$ and coupled with the constants $\left\{J_{i j}\right\}$. In principle, the qubits can be biased and coupled along any direction, $x, y$, or $z$. The chain has $N$ qubits, with a uniform ferromagnetic coupling between the nearby qubits, $J_{i j}^{z}=-J \delta_{i \pm 1, j}$, where $J>0$. Notice that hereafter we work in the $z$-basis since the probe qubit is coupled to $\sigma_{i}^{z}$-matrices of the source qubits. This can be done by means of the standard magnetic couplers [3, 4]. The frustration is created by two additional boundary qubits, which have fixed spin directions. In Fig. 1 we show configurations of seven source spins $(N=7)$ plus two boundary qubits. The left boundary qubit is always in the up-direction, and the right boundary qubit is always directed downwards. These qubits are ferromagnetically coupled to the first and to the last qubits in the Ising chain, thus creating a bias $h_{1}=-J$ applied to the first qubit and the bias $h_{N}=J$ applied to the last qubit in the chain. The other qubits have zero biases, $h_{i}=0$ at $i=2, \ldots, N-1$. In the absence of the probe qubit the lowest energy of the $N$-spin Ising chain is degenerate. All eigenstates of the source Hamiltonian $H_{S}$ with the lowest energy have one kink located between the source qubits. A kink also can be located between the first qubit in the chain and the left boundary qubit, and between the right boundary qubit and the last qubit in the chain. The possible locations of the kink, which is shown as a blue star, are presented in Fig. 1] It is known that the kink in the Ising chain behaves like a free quantum particle. The main goal of

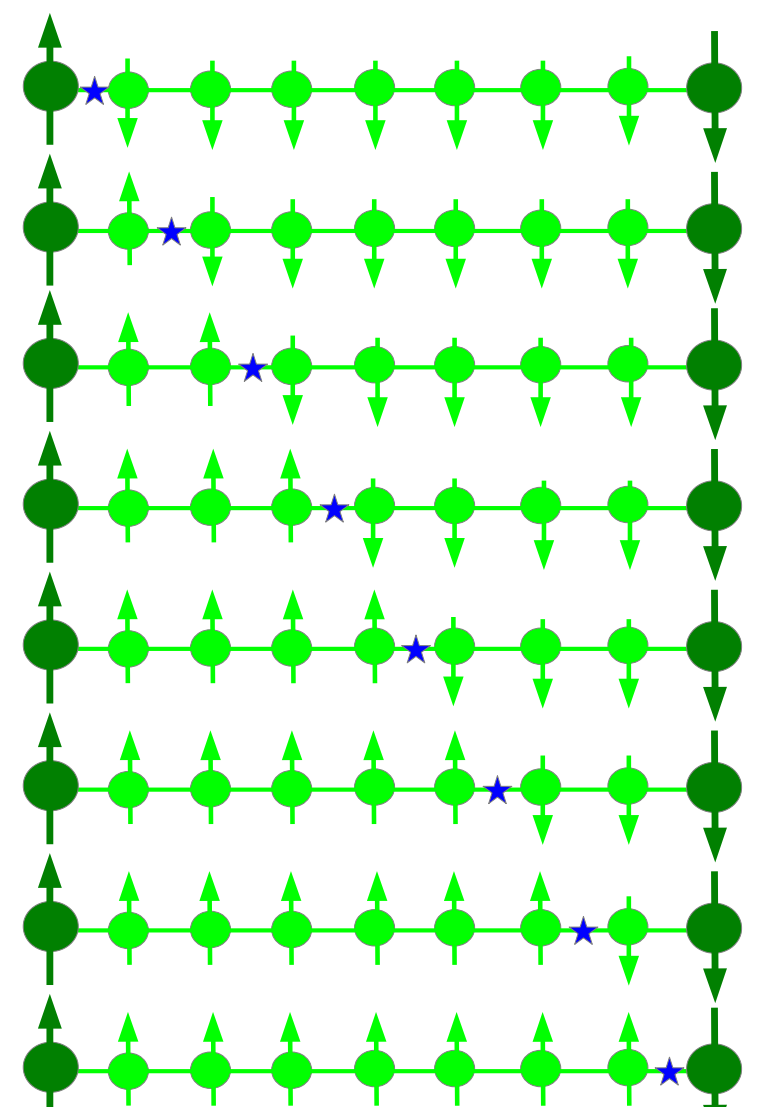

FIG. 1: Eight possible positions of a kink (shown as a blue star) in a chain with seven source qubits and two boundary spins. A left boundary qubit is always up, and a right boundary qubit is always down.

this part of the paper is to demonstrate that the QTS measurements allow us to visualize the quantum distributions of the kink in the frustrated Ising chain.

In the process of QTS tomography the lowest eigenstates of the kink's Hamiltonian are projected on the basis set formed by vectors that have a definite kink location. In particular, functions $\left|\psi_{1}\right\rangle, \ldots,\left|\psi_{N+1}\right\rangle$, where

$$
\begin{aligned}
\left|\psi_{1}\right\rangle & =\left|\downarrow_{1}, \downarrow_{2}, \ldots, \downarrow_{N-1}, \downarrow_{N}\right\rangle, \\
\left|\psi_{2}\right\rangle & =\left|\uparrow_{1}, \downarrow_{2}, \ldots, \downarrow_{N-1}, \downarrow_{N}\right\rangle, \ldots, \\
\left|\psi_{N}\right\rangle & =\left|\uparrow_{1}, \uparrow_{2}, \ldots, \uparrow_{N-1}, \downarrow_{N}\right\rangle, \\
\left|\psi_{N+1}\right\rangle & =\left|\uparrow_{1}, \uparrow_{2}, \ldots, \uparrow_{N-1}, \uparrow_{N}\right\rangle,
\end{aligned}
$$

corresponds to $N+1$ positions of the kink in the frustrated Ising chain (see Fig. 1 for the case of seven source qubits, $N=7$ ). These functions form the quantummechanical basis. We notice that the basis $\left|\psi_{l}\right\rangle$ is not complete since the high-energy states with many kinks are neglected here. Every state from the set $\left\{\left|\psi_{1}\right\rangle, \ldots,\left|\psi_{N+1}\right\rangle\right\}$ is characterized by a definite position of the kink. An arbitrary quantum state, for example, the $n$-eigenstate of the source qubits, $\left|\Psi_{n}^{\uparrow}\right\rangle \equiv\left|\Psi_{n}\right\rangle$, can 
be represented as a superposition of the basis states $\left|\psi_{l}\right\rangle$,

$$
\left|\Psi_{n}^{\uparrow}\right\rangle=\sum_{l=1}^{N+1}\left\langle\psi_{l} \mid \Psi_{n}^{\uparrow}\right\rangle\left|\psi_{l}\right\rangle .
$$

The set of amplitudes, $C_{l}^{(n)}=\left\langle\psi_{l} \mid \Psi_{n}^{\uparrow}\right\rangle$, taken as functions of the quantum number $l$, describes a wave function of the $n$-th energy state in a single-kink representation. The quantum number $l$ serves as a position of the kink in the frustrated Ising chain. Thus, the $l$-dependence of the kink amplitude $C_{l}^{(n)}$ is equivalent to the coordinate dependence of the wave function of the particle in the state corresponding to the $n$-eigenstate of the source Hamiltonian $H_{S}$. Here we have $n=0,1, \ldots,\left(2^{N}-1\right)$.

We notice that the escape rate $\Gamma_{0}(\epsilon)$ (5) is proportional to the overlap squared, $\left|\left\langle\Psi_{n}^{\uparrow} \mid \Psi_{0}^{\downarrow}\right\rangle\right|^{2}$, of the $n$-eigenstate $\left|\Psi_{n}^{\uparrow}\right\rangle$ of the source Hamiltonian $H_{S}$ and the ground state $\left|\Psi_{0}^{\downarrow}\right\rangle$ of the biased source qubits. The ground state $\left|\Psi_{0}^{\downarrow}\right\rangle$ of the left manifold can be transformed into a specific basis state $\left|\psi_{l}\right\rangle$, so that $\left|\Psi_{0}^{\downarrow}\right\rangle=\left|\psi_{l}\right\rangle$, by choosing proper couplings $J_{p i}$ between the probe and the source qubits as it is shown in Fig. 2. For example, the first state $\left|\psi_{1}\right\rangle=\left|\downarrow_{1} \ldots \downarrow_{N}\right\rangle$ can be generated if the first qubit in the chain is coupled to the probe with a positive constant $J_{p 1}=J_{p}>0$. Other source qubits are decoupled from the probe. The second basis state $\left|\psi_{2}\right\rangle=\left|\uparrow_{1} \downarrow_{2} \ldots \downarrow_{N}\right\rangle$ is created when the probe qubit is coupled to the first source qubit by negative coupling, $J_{p 1}=-J_{p}$, whereas its coupling to the second qubit is positive, $J_{p 2}=J_{p}>$ 0 . To generate the state $\left|\psi_{l}\right\rangle=\left|\uparrow_{1} \uparrow_{2} \ldots \uparrow_{l-1} \downarrow_{l} \ldots \downarrow_{N}\right\rangle$, two nearby qubits $l-1$ and $l$ should be coupled to the probe with opposite coupling strengths, $J_{p, l-1}=-J_{p}$ and $J_{p, l}=J_{p}$. The last state, $\left|\psi_{N+1}\right\rangle=\left|\uparrow_{1} \ldots \uparrow_{N}\right\rangle$, is generated when the $N$-qubit is coupled to the probe with a negative coupling strength, $J_{p N}=J_{p}$, and other source qubits do not interact with the probe.

\section{A. Quantum distribution of a kink}

In order to obtain the quantum distribution of the system over all possible positions of the kink in the Ising chain we have to measure the $l$-dependence of the function $\left|C_{l}^{(n)}\right|^{2}=\left|\left\langle\psi_{l} \mid \Psi_{n}\right\rangle\right|^{2}$. To do that, we choose a specific connection between the probe and source qubits related, for example, to the state $\left|\psi_{l}\right\rangle$, with a subsequent measurement of the escape rate $\Gamma_{0}(\epsilon)$ for all possible probe biases $\epsilon$. As the next step, we change the initial source-probe connection and repeat the measurements. Finally, we obtain the rate $\Gamma_{0}(\epsilon, l)$ as a function of the bias $\epsilon$ and the kink position, which is characterized by the number $l$ of the single-kink basis state $\left|\psi_{l}\right\rangle$. In Fig. 3 we show the normalized function $\Gamma_{0}(\epsilon, l)$ for the case of seven qubits in the chain $(N=7)$. Both figures are plotted at $\Delta_{1}=\ldots=\Delta_{N}=2 \mathrm{GHz}, J=2 \mathrm{GHz}, J_{p}=J$, with a temperature of $T=12 \mathrm{mK}$ and a MRT linewidth of $W=10 \mathrm{mK}$. Only four lowest energy levels of source
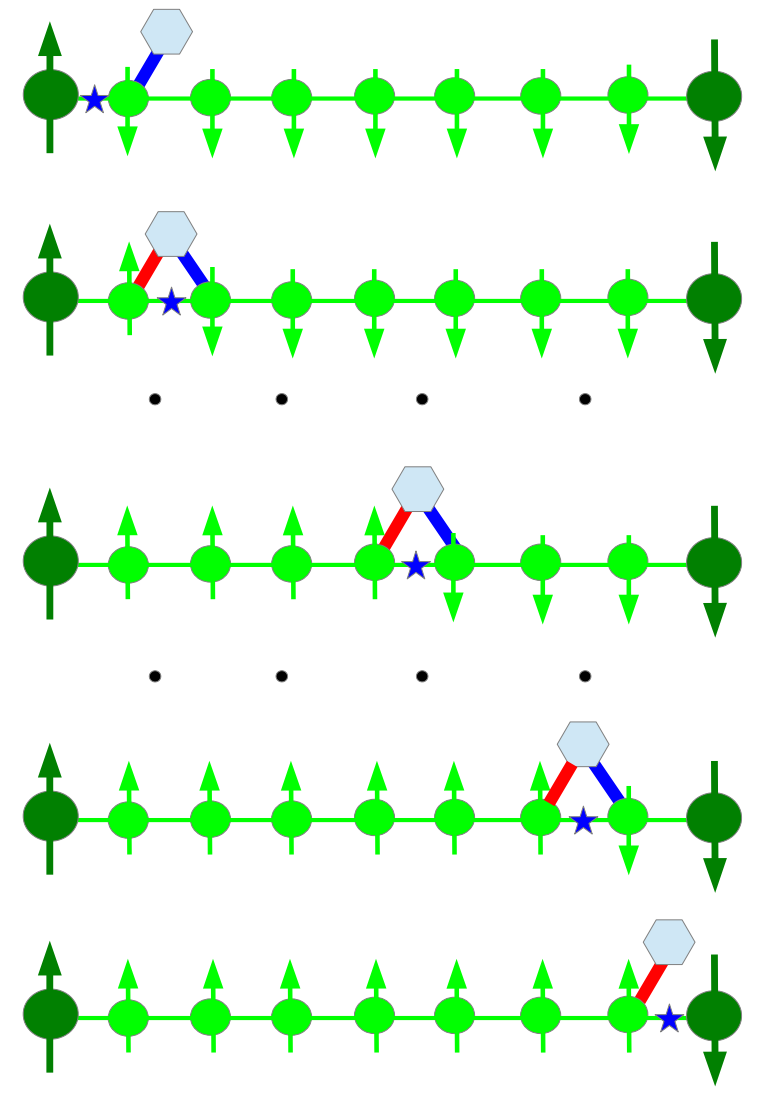

FIG. 2: A generation of basis states $\left|\psi_{l}\right\rangle$ of the left manifold by selective coupling of the probe qubit (shown as a hexagon) to the source qubits. A positive probe-source coupling $J_{p i}$ is drawn in blue, a negative $J_{p i}$ is shown in red. A blue star symbol is related to the kink location.

qubits, with $n=0,1,2,3$, are presented. In Fig. 4 we plot the QTS rate $\Gamma_{0}(\epsilon, l)$ for the chain that has 16 qubits $(N=16)$ and for the same set of parameters. This figure has a better resolution than Fig. 3. In both figures, along the $\epsilon$-axis we have the standard QTS peaks corresponding to four energy eigenstates $\left|\Psi_{n}\right\rangle$ of the source Hamiltonian $H_{S}$ (9). If we move along the other axis, we will see a dependence of the states $\left|\Psi_{n}\right\rangle$ on the kink position. The probability distributions of the kink in the frustrated chain shown in Figs. 3] and 4 are similar to the distribution of a quantum particle in a potential well.

\section{CONCLUSIONS}

We have generalized the qubit tunneling spectroscopy approach of Ref. [5] to allow performing quantum eigenstate tomography in a multi-qubit (source) system. An additional (probe) qubit, working in the incoherent regime, has to be coupled to all source qubits in all bases to make projective measurement onto an arbitrary basis state possible. A limited, but practical, version of to- 


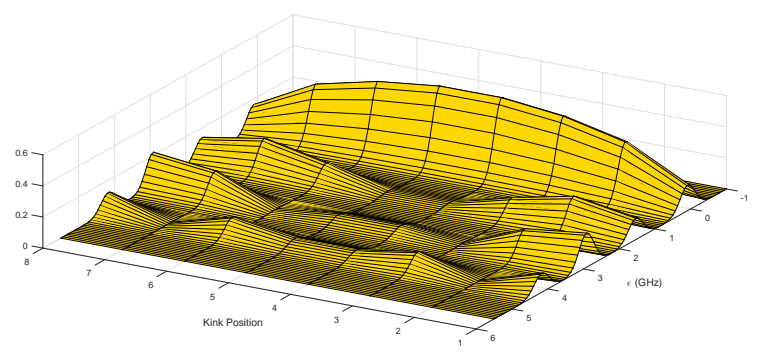

FIG. 3: Escape rate $\Gamma_{0}$ as a function of the kink position $l$ and the bias $\epsilon$ applied to the probe qubit. Here we have seven source qubits. As in the case of a free particle in the potential well, the ground state of the kink has a maximum in the middle of the chain where the first excited state has a node. The second and third excited states have two and three nodes, respectively.

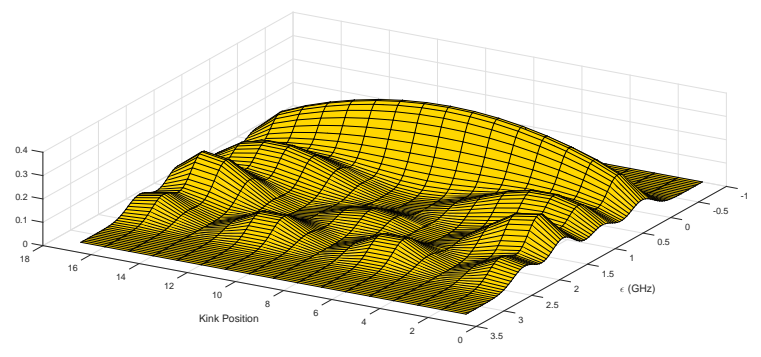

FIG. 4: the QTS rate $\Gamma_{0}(\epsilon, l)$ reflects the quantum distribution of a kink position $l$ in the 16-qubit Ising chain for four lowest energy eigenstates. The peak with the energy $\epsilon \sim 0$ corresponds to the ground state of the kink (particle). The next peak, with $\epsilon \sim 1 \mathrm{GHz}$, is related to the first excited state having one node, at $l=8$, in the wave function. The wave function of the next state, with the energy $\epsilon \sim 1.5 \mathrm{GHz}$, has two nodes, at $l=6$ and $l=12$. The last state shown in the picture has the energy $\epsilon \sim 2.25 \mathrm{GHz}$. This state is described by the wave function having three nodes located at $l=4$, $l=9$, and $l=14$.

mography is described with an example of a single kink in a frustrated Ising chain. The lowest energy eigenstates of this system is equivalent to those of a free quantum particle confined in a potential well. We have calculated the incoherent tunneling rate of the system and shown that its peak values correspond to the modulus squared of the overlap of the eigenstates and a preselected basis state which is related to a kink position.

\section{Acknowledgements}

We are thankful to Professor Alexander Omelyanchouk for collaboration and support in early days of D-Wave Systems. We are grateful to Chris Rich for helpful discussions and Fiona Hanington for critical reading of the paper.

\section{Appendix A: Derivation of the master equation}

The system of $N$ source qubits and one probe qubit is described by the Hamiltonian $H_{0}$ defined by Eqs. (1) and (2). The probe qubit is working in a regime of incoherent tunneling between its wells. The tunneling is introduced by the small tunneling amplitude $\Delta_{p}$ in the Hamiltonian $H_{0}$. We also take into account an interaction of the probe qubit with its dissipative environment, which is described by the variable $Q_{p}$. Weak coupling of source qubits to their environments is omitted here. This coupling contributes to the width of MRT lines of the probe qubit[7]. The main contribution to the linewidth, however, is given by the low-frequency bath directly coupled to the probe. The total Hamiltonian $H$ of the source-probe system coupled to the probe qubit bath has the form

$$
H=H_{0}-Q_{p} \sigma_{p}^{z}+H_{B}
$$

where $H_{B}$ is the free Hamiltonian of the bath, and $\sigma_{p}^{x}$ is the Pauli matrix responsible for the flipping of the probe qubits between states $\left|\downarrow_{p}\right\rangle$ and $\left|\uparrow_{p}\right\rangle, \sigma_{p}^{x}=$ $\left|\uparrow_{p}\right\rangle\left\langle\downarrow_{p}|+| \downarrow_{p}\right\rangle\left\langle\uparrow_{p}\right|$.

The bath can be represented as a sum of independent harmonic oscillators [11, 12] with the Hamiltonian

$$
H_{B}=\sum_{k}\left(\frac{p_{k}^{2}}{2 m_{k}}+\frac{m_{k} \omega_{k}^{2} x_{k}^{2}}{2}\right)
$$

The $k$-th oscillator in the bath is characterized by position $x_{k}$, momentum $p_{k}$, mass $m_{k}$ and positive frequency $\omega_{k}$. The bath operator $Q_{p}$ in Eq. A1 is given by the formula

$$
Q_{p}=\sum_{k} m_{k} \omega_{k}^{2} z_{k p} x_{k}
$$

A constant $z_{k p}$ determines the strength of coupling between the probe qubit and the $k$-mode of the bath. The Hamiltonian $H$ (A1) can be written as

$$
\begin{array}{r}
H=H_{S}^{\uparrow} \otimes\left|\uparrow_{p}\right\rangle\left\langle\uparrow_{p}\left|+H_{S}^{\downarrow} \otimes\right| \downarrow_{p}\right\rangle\left\langle\downarrow_{p}\right|-\Delta_{p} \sigma_{p}^{x}+ \\
\sum_{k} \frac{p_{k}^{2}}{2 m_{k}}+\sum_{k} \frac{m_{k} \omega_{k}^{2}}{2}\left(x_{k}-z_{k p} \sigma_{p}^{z}\right)^{2} .
\end{array}
$$

Here we have omitted the constant term. The unitary transformation

$$
\begin{array}{r}
U_{p}=e^{-i \xi_{p} \sigma_{p}^{z}}= \\
1+\left(e^{-i \xi_{p}}-1\right)\left|\downarrow_{p}\right\rangle\left\langle\downarrow_{p}\left|+\left(e^{i \xi_{p}}-1\right)\right| \uparrow_{p}\right\rangle\left\langle\uparrow_{p}\right|,
\end{array}
$$

applied to the Hamiltonian $H$ turns this operator to the form

$$
\begin{array}{r}
H^{\prime}=U_{p}^{\dagger} H U_{p}=\sum_{k}\left(\frac{p_{k}^{2}}{2 m_{k}}+\frac{m_{k} \omega_{k}^{2} x_{k}^{2}}{2}\right)+ \\
H_{S}^{\uparrow} \otimes\left|\uparrow_{p}\right\rangle\left\langle\uparrow_{p}\left|+H_{S}^{\downarrow} \otimes\right| \downarrow_{p}\right\rangle\left\langle\downarrow_{p}\right|- \\
\Delta_{p} e^{2 i \xi_{p}}\left|\downarrow_{p}\right\rangle\left\langle\uparrow_{p}\left|-\Delta_{p} e^{-2 i \xi_{p}}\right| \uparrow_{p}\right\rangle\left\langle\downarrow_{p}\right| .
\end{array}
$$


Here $\xi_{p}=\sum_{k} z_{k p} p_{k}$ is a stochastic phase produced by the bath. We notice that this phase appears only at the tunneling terms.

The source-probe Hamiltonian has eigenstates $\left|\Psi_{\mu}\right\rangle$ defined by the following equations:

$$
\left(H_{S}^{\uparrow} \otimes\left|\uparrow_{p}\right\rangle\left\langle\uparrow_{p}\left|+H_{S}^{\downarrow} \otimes\right| \downarrow_{p}\right\rangle\left\langle\downarrow_{p}\right|\right)\left|\Psi_{\mu}\right\rangle=E_{\mu}\left|\Psi_{\mu}\right\rangle,(
$$

where $\mu \in\left\{0,1, \ldots,\left(2^{N+1}-1\right)\right\}$. We notice that the set of eigenstates $\left\{\left|\Psi_{\mu}\right\rangle\right\}$ contains two subsets - one, which is related to the up-state of the probe qubit, and another, which is related to the down-state of the probe: $\left\{\left|\Psi_{\mu}\right\rangle\right\}=\left\{\left|\Psi_{n}^{\uparrow}\right\rangle \otimes\left|\uparrow_{p}\right\rangle,\left|\Psi_{m}^{\downarrow}\right\rangle \otimes\left|\downarrow_{p}\right\rangle\right.$. Here the eigenstates $\left|\Psi_{n}^{\uparrow}\right\rangle$ and $\left|\Psi_{m}^{\downarrow}\right\rangle$ can be found from Eqs. (3). The indices $m$ and $n$ run over $2^{N}$ states: $m, n \in\left\{0,1, \ldots,\left(2^{N}-1\right)\right\}$. The eigenenergies $\left\{E_{\mu}\right\}$ also have two subsets: $\left\{E_{\mu}\right\}=$ $\left\{E_{n}^{\uparrow}, E_{m}^{\downarrow}+\epsilon\right\}$, with $\epsilon$ being the bias applied to the probe qubit.

Following the approach proposed in Ref. [13] and developed in Ref. [14] we introduce a time-dependent Heisenberg operator $\rho_{\mu \nu}$ of the source-probe system,

$$
\rho_{\mu \nu}=\left(\left|\Psi_{\mu}\right\rangle\left\langle\Psi_{\nu}\right|\right)(t)
$$

In the Heisenberg representation the total Hamiltonian $H$ (A6) is given by the formula

$$
H=\sum_{\mu} E_{\mu} \rho_{\mu \mu}-\sum_{\mu \neq \nu} Q_{\mu \nu} \rho_{\mu \nu}+H_{B},
$$

with the bath operator

$$
\begin{array}{r}
Q_{\mu \nu}=\Delta_{p} e^{2 i \xi_{p}}\left\langle\Psi_{\mu} \mid \downarrow_{p}\right\rangle\left\langle\uparrow_{p} \mid \Psi_{\nu}\right\rangle+ \\
\Delta_{p} e^{-2 i \xi_{p}}\left\langle\Psi_{\mu} \mid \uparrow_{p}\right\rangle\left\langle\downarrow_{p} \mid \Psi_{\nu}\right\rangle .
\end{array}
$$

Here we drop the prime sign in the Hamiltonian $H$ (A6). The diagonal elements of the Hamiltonian (A8) are determined by the eigenenergies $E_{\mu}$ of the system-probe Hamiltonian $H_{0}$ (2). The bath Hamiltonian $H_{B}$ is defined by Eq. A22). The operator $\rho_{\mu \nu}$ obeys the Heisenberg equation

$$
\dot{\rho}_{\mu \nu}=i \omega_{\mu \nu} \rho_{\mu \nu}+i \sum_{\mu^{\prime}}\left(Q_{\nu \mu^{\prime}} \rho_{\mu \mu^{\prime}}-Q_{\mu^{\prime} \mu} \rho_{\mu^{\prime} \nu}\right),
$$

with $\omega_{\mu \nu}=E_{\mu}-E_{\nu}$. Using the approach developed in Refs. [13, 14] we derive a set of equations for the qubit operators averaged over fluctuations of the free bath,

$$
\begin{array}{r}
\left\langle\dot{\rho}_{\mu \nu}\right\rangle-i \omega_{\mu \nu}\left\langle\rho_{\mu \nu}\right\rangle= \\
-\int_{0}^{t} d t_{1}\left\langle Q_{\nu \mu^{\prime}}^{(0)}(t) Q_{\mu^{\prime \prime} \nu^{\prime \prime}}^{(0)}\left(t_{1}\right)\right\rangle\left\langle\rho_{\mu \mu^{\prime}}(t) \rho_{\mu^{\prime \prime} \nu^{\prime \prime}}\left(t_{1}\right)\right\rangle \\
+\int_{0}^{t} d t_{1}\left\langle Q_{\mu^{\prime \prime} \nu^{\prime \prime}}^{(0)}\left(t_{1}\right) Q_{\nu \mu^{\prime}}^{(0)}(t)\right\rangle\left\langle\rho_{\mu^{\prime \prime} \nu^{\prime \prime}}\left(t_{1}\right) \rho_{\mu \mu^{\prime}}(t)\right\rangle \\
+\int_{0}^{t} d t_{1}\left\langle Q_{\mu^{\prime} \mu}^{(0)}(t) Q_{\mu^{\prime \prime} \nu^{\prime \prime}}^{(0)}\left(t_{1}\right)\right\rangle\left\langle\rho_{\mu^{\prime} \nu}(t) \rho_{\mu^{\prime \prime} \nu^{\prime \prime}}\left(t_{1}\right)\right\rangle \\
-\int_{0}^{t} d t_{1}\left\langle Q_{\mu^{\prime \prime} \nu^{\prime \prime}}^{(0)}\left(t_{1}\right) Q_{\mu^{\prime} \mu}^{(0)}(t)\right\rangle\left\langle\rho_{\mu^{\prime \prime} \nu^{\prime \prime}}\left(t_{1}\right) \rho_{\mu^{\prime} \nu}(t)\right\rangle .
\end{array}
$$

An operator $Q_{\mu \nu}^{(0)}(t)$ is defined by Eq. (A99) where the stochastic phases $\xi_{p}$ are replaced by their unperturbed values $\xi_{p}^{(0)}=\sum_{k} z_{k p} p_{k}^{(0)}$ that have free Heisenberg operators $p_{k}^{(0)}$ of the bath. A time evolution of the free bath operators is determined by the Hamiltonian $H_{B}$ (A2). We also assume that there are sums over repeated indices $\mu^{\prime}, \mu^{\prime \prime}, \nu^{\prime \prime}$ in the right-hand side of Eq. (A11). The free bath variables $Q_{\mu \nu}^{(0)}(t)$ are nonlinear functions of the Gaussian bath operators $\xi_{p}^{(0)}$. Therefore, nonMarkovian equations (A11) are valid at the small systembath coupling only. We consider a regime of incoherent tunneling for the probe qubit. This regime takes place at the small tunneling amplitude $\Delta_{p}$. It follows from Eq. (A9) that the small $\Delta_{p}$ is related to the small system-bath interaction. Within the perturbation theory in terms of the parameter $\Delta_{p}$ we assume that in Eq. A11 the correlation functions of the qubit operators, such as $\left\langle\rho_{\mu \mu^{\prime}}(t) \rho_{\mu^{\prime \prime} \nu^{\prime \prime}}\left(t_{1}\right)\right\rangle$, can be calculated using the free evolution equations. It is convenient to reduce the operator $\rho_{\mu^{\prime \prime} \nu^{\prime \prime}}\left(t_{1}\right)$ to the operator $\rho_{\mu^{\prime \prime} \nu^{\prime \prime}}(t)$ in such a way that

$$
\rho_{\mu^{\prime \prime} \nu^{\prime \prime}}\left(t_{1}\right)=e^{-i \omega_{\mu^{\prime \prime} \nu^{\prime \prime}}\left(t-t_{1}\right)} \rho_{\mu^{\prime \prime} \nu^{\prime \prime}}(t),
$$

so that the correlator is given by the equation

$$
\left\langle\rho_{\mu \mu^{\prime}}(t) \rho_{\mu^{\prime \prime} \nu^{\prime \prime}}\left(t_{1}\right)\right\rangle=\delta_{\mu^{\prime} \mu^{\prime \prime}} e^{-i \omega_{\mu^{\prime \prime} \nu^{\prime \prime}}\left(t-t_{1}\right)} \rho_{\mu \nu^{\prime \prime}}(t),
$$

where $\delta_{\mu^{\prime} \mu^{\prime \prime}}$ is the Kronecker delta.

A diagonal element $P_{\mu}=\left\langle\rho_{\mu \mu}\right\rangle$ of the averaged matrix $\left\langle\rho_{\mu \nu}\right\rangle$ defines the probability to find the source-probe system in the state $\left|\Psi_{\mu}\right\rangle$. It follows from Eqs. A11 that these probabilities are governed by the set of master equations,

$$
\dot{P}_{\mu}+\Gamma_{\mu} P_{\mu}=\sum_{\nu} \Gamma_{\mu \nu} P_{\nu}
$$

Here $\Gamma_{\mu}=\sum_{\nu} \Gamma_{\nu \mu}$ is a relaxation rate and $\Gamma_{\mu \nu}$ is a relaxation matrix defined by the equation

$$
\Gamma_{\mu \nu}=\int_{0}^{t} d t_{1}\left\langle Q_{\nu \mu}^{(0)}(t) Q_{\mu \nu}^{(0)}\left(t_{1}\right)\right\rangle e^{-i \omega_{\mu \nu}\left(t-t_{1}\right)}+h . c(\mathrm{~A} 1
$$

The bath operator $Q_{\mu \nu}^{(0)}(t)$ is given by Eq. (A9) where $\xi_{p}^{(0)}$ is a free Gaussian operator, $\xi_{p}^{(0)}=\sum_{k} z_{k p} p_{k}^{(0)}$. The commutator and the correlation function of these Heisenberg operators taken at different moments of time are determined by the following expressions

$$
\begin{array}{r}
\frac{1}{2}\left[\xi_{p}^{(0)}(t), \xi_{p}^{(0)}\left(t^{\prime}\right)\right]_{-}=-i \sum_{k} \frac{m_{k} \omega_{k} z_{k p}^{2}}{2} \sin \omega_{k}\left(t-t^{\prime}\right)= \\
-i \int \frac{d \omega}{2 \pi} \frac{\chi_{p}^{\prime \prime}(\omega)}{\omega^{2}} \sin \omega\left(t-t^{\prime}\right), \\
\left\langle\frac{1}{2}\left[\xi_{p}^{(0)}(t), \xi_{p}^{(0)}\left(t^{\prime}\right)\right]_{+}\right\rangle= \\
\sum_{k} \frac{m_{k} \omega_{k} z_{k p}^{2}}{2} \operatorname{coth}\left(\frac{\omega_{k}}{2 T}\right) \cos \omega_{k}\left(t-t^{\prime}\right)=
\end{array}
$$




$$
\int \frac{d \omega}{2 \pi} \frac{S_{p}(\omega)}{\omega^{2}} \cos \omega\left(t-t^{\prime}\right)
$$

with $T$ being the equilibrium temperature of the free bath. The dissipative properties of the bath are defined by the imaginary part of its susceptibility $\chi_{p}^{\prime \prime}(\omega)$ and by the spectrum $S_{p}(\omega)$. They are described by the following formulas

$$
\begin{array}{r}
\chi_{p}^{\prime \prime}(\omega)=\pi \sum_{k} \frac{m_{k} \omega_{k}^{3} z_{k p}^{2}}{2}\left[\delta\left(\omega-\omega_{k}\right)-\delta\left(\omega+\omega_{k}\right)\right], \\
S_{p}(\omega)=\chi_{p}^{\prime \prime}(\omega) \operatorname{coth}\left(\frac{\omega}{2 T}\right)= \\
\pi \sum_{k} \frac{m_{k} \omega_{k}^{3} z_{k p}^{2}}{2} \operatorname{coth}\left(\frac{\omega_{k}}{2 T}\right) \times \\
{\left[\delta\left(\omega-\omega_{k}\right)+\delta\left(\omega+\omega_{k}\right)\right] .}
\end{array}
$$

For the correlator of free variables $Q_{\mu \nu}^{(0)}$ of the bath we obtain

$$
\left\langle Q_{\nu \mu}^{(0)}(t) Q_{\mu \nu}^{(0)}\left(t^{\prime}\right)\right\rangle=\Delta_{\mu \nu}^{2} \Phi_{p}\left(t-t^{\prime}\right),
$$

where the prefactor $\Delta_{\mu \nu}^{2}$ is defined as

$$
\begin{array}{r}
\Delta_{\mu \nu}^{2}=\Delta_{p}^{2}\left(\left|\left\langle\Psi_{\mu} \mid \downarrow_{p}\right\rangle\left\langle\uparrow_{p} \mid \Psi_{\nu}\right\rangle\right|^{2}+\right. \\
\left.\left|\left\langle\Psi_{\mu} \mid \uparrow_{p}\right\rangle\left\langle\downarrow_{p} \mid \Psi_{\nu}\right\rangle\right|^{2}\right) .
\end{array}
$$

The characteristic functional of the bath is given by the formula:

$$
\begin{array}{r}
\Phi_{p}\left(t-t^{\prime}\right)=\left\langle e^{2 i \xi_{p}^{(0)}(t)} e^{-2 i \xi_{p}^{(0)}\left(t^{\prime}\right)}\right\rangle= \\
\exp \left[-4 \int \frac{d \omega}{2 \pi} S_{p}(\omega) \frac{1-\cos \omega\left(t-t^{\prime}\right)}{\omega^{2}}-\right. \\
\left.4 i \int \frac{d \omega}{2 \pi} \chi_{p}^{\prime \prime}(\omega) \frac{\sin \omega\left(t-t^{\prime}\right)}{\omega^{2}}\right] .
\end{array}
$$

The heat bath acting on the probe qubit may have both, low-frequency and high-frequency, components [15]. In this case the dissipative function $\chi_{p}^{\prime \prime}(\omega)$ is represented as a sum of the low-frequency susceptibility, $\chi_{L F}^{\prime \prime}(\omega)$, and the high-frequency function $\chi_{H F}^{\prime \prime}(\omega): \chi_{p}^{\prime \prime}(\omega)=\chi_{L F}^{\prime \prime}(\omega)+$ $\chi_{H F}^{\prime \prime}(\omega)$. The functional $\Phi_{p}\left(t, t^{\prime}\right)$ is equal to the product of the low-frequency and high-frequency parts: $\Phi_{p}(\tau)=$ $\Phi_{L F}(\tau) \Phi_{H F}(\tau)$. Here, the low-frequency factor is determined by the formula

$$
\Phi_{L F}(\tau)=e^{-i \epsilon_{p} \tau} \exp \left(-\frac{W^{2} \tau^{2}}{2}\right),
$$

with the reorganization energy $\epsilon_{p}$ and with the width $W$ defined by the following equations,

$$
\begin{array}{r}
\epsilon_{p}=4 \int \frac{d \omega}{2 \pi} \frac{\chi_{L F}^{\prime \prime}(\omega)}{\omega}, \\
W^{2}=4 \int \frac{d \omega}{2 \pi} S_{L F}(\omega)=2 T \epsilon_{p} .
\end{array}
$$

The high-frequency noise acting on the probe qubit is usually described by the Ohmic spectral density

$$
\chi_{H F}^{\prime \prime}(\omega)=\eta \omega e^{-|\omega| / \omega_{c}},
$$

where $\eta$ is a small dimensionless coupling constant and $\omega_{c}$ is the cutoff frequency [12]. In this case the highfrequency factor $\Phi_{H F}(\tau)$ of the functional $\Phi_{p}(\tau)$ is given by the expression

$$
\Phi_{H F}(\tau)=\left[\frac{1}{1+i \omega_{c} \tau} \frac{\pi T \tau}{\sinh (\pi T \tau)}\right]^{4 \eta / \pi} .
$$

The relaxation matrix (A24) can be written as

$$
\begin{aligned}
\Gamma_{\mu \nu}=\Delta_{\mu \nu}^{2} & \int_{0}^{\infty} d \tau e^{-i\left(\omega_{\mu \nu}+\epsilon_{p}\right) \tau} e^{-W^{2} \tau^{2} / 2} \times \\
& {\left[\frac{1}{1+i \omega_{c} \tau} \frac{\pi T \tau}{\sinh (\pi T \tau)}\right]^{4 \eta / \pi}+\text { h.c. } }
\end{aligned}
$$

A more comprehensive description of the dissipative dynamics of the open quantum system has been carried out in Ref. [16]. We notice that in the case of the very weak coupling of the slow probe qubit to the high-frequency bath, when $4 \eta / \pi \ll 1$, the relaxation matrix is given by the Marcus formula [9],

$$
\Gamma_{\mu \nu}=\Delta_{\mu \nu}^{2} \sqrt{\frac{2 \pi}{W^{2}}} \exp \left[-\frac{\left(\omega_{\mu \nu}+\epsilon_{p}\right)^{2}}{2 W^{2}}\right]
$$

For indices $\mu$ and $\nu$ we have two possible cases:

(a) $\left|\Psi_{\mu}\right\rangle=\left|\Psi_{n}^{\uparrow}\right\rangle \otimes\left|\uparrow_{p}\right\rangle,\left|\Psi_{\nu}\right\rangle=\left|\Psi_{m}^{\downarrow}\right\rangle \otimes\left|\downarrow_{p}\right\rangle$,

and

(b) $\left|\Psi_{\mu}\right\rangle=\left|\Psi_{m}^{\downarrow}\right\rangle \otimes\left|\downarrow_{p}\right\rangle,\left|\Psi_{\nu}\right\rangle=\left|\Psi_{n}^{\uparrow}\right\rangle \otimes\left|\uparrow_{p}\right\rangle$.

These cases correspond to two sets of eigensenergies and frequencies:

(a) $E_{\mu}=E_{n}^{\uparrow}, E_{\nu}=E_{m}^{\downarrow}+\epsilon, \omega_{\mu \nu}=E_{n}^{\uparrow}-E_{m}^{\downarrow}-\epsilon$,

and

(b) $E_{\mu}=E_{m}^{\downarrow}+\epsilon, E_{\nu}=E_{n}^{\uparrow}, \omega_{\mu \nu}=E_{m}^{\downarrow}-E_{n}^{\uparrow}+\epsilon$.

For these two sets we obtain the following relaxation matrices:

$$
\begin{array}{r}
\text { (a) } \Gamma_{\mu \nu}^{(a)}=\Delta_{p}^{2}\left|\left\langle\Psi_{n}^{\uparrow} \mid \Psi_{m}^{\downarrow}\right\rangle\right|^{2} \times \\
\sqrt{\frac{2 \pi}{W^{2}}} \exp \left[-\frac{\left(E_{n}^{\uparrow}-E_{m}^{\downarrow}-\epsilon+\epsilon_{p}\right)^{2}}{2 W^{2}}\right], \\
\text { (b) } \Gamma_{\mu \nu}^{(b)}=\Delta_{p}^{2}\left|\left\langle\Psi_{n}^{\uparrow} \mid \Psi_{m}^{\downarrow}\right\rangle\right|^{2} \times \\
\sqrt{\frac{2 \pi}{W^{2}}} \exp \left[-\frac{\left(E_{n}^{\uparrow}-E_{m}^{\downarrow}-\epsilon-\epsilon_{p}\right)^{2}}{2 W^{2}}\right] .
\end{array}
$$

If we start the QTS experiment with the probe qubit being in its $\left|\downarrow_{p}\right\rangle$ state and allow the qubit to tunnel into 
the $\left|\uparrow_{p}\right\rangle$ state, the situation is described by the master equation (A12) where $P_{\mu} \equiv P_{m}$ is the probability to find the system in state $\left|\Psi_{\mu}\right\rangle=\left|\Psi_{m}^{\downarrow}\right\rangle \otimes\left|\downarrow_{p}\right\rangle$. The system tunnels into the state $\left|\Psi_{\nu}\right\rangle=\left|\Psi_{n}^{\uparrow}\right\rangle \otimes\left|\uparrow_{p}\right\rangle$, so that here we have the case (b) described by the relaxation matrix $\Gamma_{\mu \nu}^{(b)} \equiv \Gamma_{m n}$ defined in Eq. (A24). The probability to find the system in the state $\left|\Psi_{\nu}\right\rangle$ is given by the variable $P_{\nu} \equiv P_{n}$. It follows from Eq. (A12) that the escape rate $\Gamma_{\mu}$ is determined by the transposed matrix $\Gamma_{\nu \mu}$, since $\Gamma_{\mu}=\sum_{\nu} \Gamma_{\nu \mu}$. In our case the matrix $\Gamma_{\nu \mu}$ is described by the case $(a)$, so that the relaxation rate $\Gamma_{\mu}$ is given by the formula

$$
\Gamma_{\mu} \equiv \Gamma_{m}=\Delta_{p}^{2} \sum_{n}\left|\left\langle\Psi_{n}^{\uparrow} \mid \Psi_{m}^{\downarrow}\right\rangle\right|^{2} \times
$$

$$
\sqrt{\frac{2 \pi}{W^{2}}} \exp \left[-\frac{\left(E_{n}^{\uparrow}-E_{m}^{\downarrow}-\epsilon+\epsilon_{p}\right)^{2}}{2 W^{2}}\right]
$$

As a result, the time evolution of the probability $P_{m}$ to find the source-probe system in the state $\left|\Psi_{m}^{\downarrow}\right\rangle \otimes\left|\downarrow_{p}\right\rangle$ is governed by the equation

$$
\dot{P}_{m}+\Gamma_{m} P_{m}=\sum_{n} \Gamma_{m n} P_{n}
$$

where $P_{n}$ is the probability for the system to be in state $\left|\Psi_{n}^{\uparrow}\right\rangle \otimes\left|\uparrow_{p}\right\rangle$.
[1] J. Q. You and Franco Nori, Superconducting Circuits and Quantum Information, Phys. Today 58 (11), 42 (2005).

[2] M.W. Johnson, M. H. S. Amin, S. Gildert, T. Lanting, F. Hamze, N. Dickson, R. Harris, A. J. Berkley, J. Johansson, P. Bunyk, E. M. Chapple, C. Enderud, J. P. Hilton, K. Karimi, E. Ladizinsky, N. Ladizinsky, T. Oh, I. Perminov, C. Rich, M. C. Thom, E. Tolkacheva, C. J. S. Truncik, S. Uchaikin, J. Wang, B. Wilson, and G. Rose, Quantum annealing with manufactured spins, Nature 473, 194 (2011).

[3] R. Harris, J. Johansson, A. J. Berkley, M. W. Johnson, T. Lanting, Siyuan Han, P. Bunyk, E. Ladizinsky, T. Oh, I. Perminov, E. Tolkacheva, S. Uchaikin, E. M. Chapple, C. Enderud, C. Rich, M. Thom, J. Wang, B. Wilson, and G. Rose, Experimental demonstration of a robust and scalable flux qubit, Phys. Rev. B 81, 134510 (2010).

[4] R. Harris, M. W. Johnson, T. Lanting, A. J. Berkley, J. Johansson, P. Bunyk, E. Tolkacheva, E. Ladizinsky, N. Ladizinsky, T. Oh, F. Cioata, I. Perminov, P. Spear, C. Enderud, C. Rich, S. Uchaikin, M. C. Thom, E. M. Chapple, J. Wang, B. Wilson, M. H. S. Amin, N. Dickson, K. Karimi, B. Macready, C. J. S. Truncik, and G. Rose Experimental investigation of an eight-qubit unit cell in a superconducting optimization processor, Phys. Rev. B 82, 024511 (2010).

[5] A. J. Berkley, A. J. Przybysz, T. Lanting, R. Harris, N. Dickson, F. Altomare, M. H. Amin, P. Bunyk, C. Enderud, E. Hoskinson, M. W. Johnson, E. Ladizinsky, R. Neufeld, C. Rich, A. Yu. Smirnov, E. Tolkacheva, S. Uchaikin, and A. B. Wilson, Tunneling spectroscopy using a probe qubit, Phys. Rev. B 87, 020502 (2013).

[6] H. Wang, S. Aschab, and Franco Nori, Quantum algorithm for obtaining the energy spectrum of a physical system, Phys. Rev. A 85, 062304 (2012).

[7] T. Lanting, A.J. Przybysz, A.Yu. Smirnov, F.M. Spedalieri, M.H. Amin, A.J. Berkley, R. Harris, F. Altomare, S. Boixo, P. Bunyk, N. Dickson, C. Enderud, J.P. Hilton, E. Hoskinson, M.W. Johnson, E. Ladizinsky, N. Ladizinsky, R. Neufeld, T. Oh, I. Perminov, C. Rich, M.C. Thom, E. Tolkacheva, S. Uchaikin, A.B. Wil- son, and G. Rose, Entanglement in a quantum annealing processor, Phys. Rev. X 4, 021041 (2014)

[8] A.J. Leggett, Macroscopic quantum systems and the quantum theory of measurement, Prog. Theor. Phys. Supplement, 69, 80 (1980).

[9] M.H.S. Amin and D.V. Averin, Macroscopic Resonant Tunneling in the Presence of Low Frequency Noise, Phys. Rev. Lett. 100, 197001 (2008)

[10] R. Harris, M.W. Johnson, S. Han, A.J. Berkley, J. Johansson, P. Bunyk, E. Ladizinsky, S. Govorkov, M.C. Thom, S. Uchaikin, B. Bumble, A. Fung, A. Kaul, A. Kleinsasser, M.H.S. Amin, D.V. Averin, Probing noise in flux qubits via macroscopic resonant tunneling, Phys. Rev. Lett. 101, 117003 (2008).

[11] V. B. Magalinskii, Dynamical model in the theory of the Brownian motion, Zh. Eksp. Teor. Fiz. 36 , 1942 (1959) [Sov. Phys. JETP 9 , 1381 (1959)].

[12] A.J. Leggett, S. Chakravarty, A.T. Dorsey, M.P.A. Fisher, A. Garg, and W. Zwerger, Dynamics of the dissipative two-state system, Rev. Mod. Phys. 59, 1 (1987)

[13] G.F. Efremov and A.Yu. Smirnov, Contribution to the microscopic theory of the fluctuations of a quantum system interacting with a Gaussian thermostat, Zh. Eksp. Teor. Fiz. 80, 1071 (1981) [Sov. Phys. JETP 53, 547 (1981)].

[14] A.Yu. Smirnov, L.G. Mourokh, and Franco Nori, Kinetics of proton pumping in cytochrome c oxidase, Journal of Chemical Physics 130, 235105 (2009).

[15] T. Lanting, M.H.S. Amin, M.W. Johnson, F. Altomare, A.J. Berkley, S. Gildert, R. Harris, J. Johansson, P. Bunyk, E. Ladizinsky, E. Tolkacheva, and D.V. Averin, Probing high-frequency noise with macroscopic resonant tunneling, Phys. Rev. B 83, 180502 (2011).

[16] S. Boixo, V.N. Smelyanskiy, A. Shabani, S.V. Isakov, M. Dykman, V.S. Denchev, M.H. Amin, A.Yu. Smirnov, M. Mohseni, and H. Neven, Computational multiqubit tunnelling in programmable quantum annealers, Nature Communications 7,10327 (2016). 